\title{
Heaps and Chains: Is the Chaining Argument for parity a sorites?
}

Article

Published Version

Elson, L. (2014) Heaps and Chains: Is the Chaining Argument for parity a sorites? Ethics, 124 (3). pp. 557-571. ISSN 00141704 doi: https://doi.org/10.1086/674844 Available at https://centaur.reading.ac.uk/39559/

It is advisable to refer to the publisher's version if you intend to cite from the work. See Guidance on citing.

Published version at: http://www.jstor.org/stable/10.1086/674844 http://www.jstor.org/stable/info/10.1086/674844 To link to this article DOI: http://dx.doi.org/10.1086/674844

Publisher: The University of Chicago Press

All outputs in CentAUR are protected by Intellectual Property Rights law, including copyright law. Copyright and IPR is retained by the creators or other copyright holders. Terms and conditions for use of this material are defined in the End User Agreement.

\section{www.reading.ac.uk/centaur}

\section{CentAUR}

Central Archive at the University of Reading

Reading's research outputs online 


\section{CHICAGO JOURNALS}

Heaps and Chains: Is the Chaining Argument for Parity a Sorites?

Author(s): Luke Elson

Source: Ethics, Vol. 124, No. 3 (April 2014), pp. 557-571

Published by: The University of Chicago Press

Stable URL: http://www.jstor.org/stable/10.1086/674844

Accessed: 11/04/2014 10:38

Your use of the JSTOR archive indicates your acceptance of the Terms \& Conditions of Use, available at

http://www.jstor.org/page/info/about/policies/terms.jsp

JSTOR is a not-for-profit service that helps scholars, researchers, and students discover, use, and build upon a wide range of content in a trusted digital archive. We use information technology and tools to increase productivity and facilitate new forms of scholarship. For more information about JSTOR, please contact support@ jstor.org. 


\title{
Heaps and Chains: Is the Chaining Argument for Parity a Sorites?*
}

\author{
Luke Elson
}

I argue that the Ruth Chang's Chaining Argument for her parity view of value incomparability trades illicitly on the vagueness of the predicate is comparable with'. Chang is alert to this danger and argues that the predicate is not vague, but this defense does not succeed. The Chaining Argument also faces a dilemma. The predicate is either vague or precise. If it is vague, then the argument is most plausibly a sorites. If it is precise, then the argument is either question begging or dialectically ineffective. I argue that no chaining-type argument can succeed.

Ruth Chang defends the strikingly original claim that there is a fourth value relation of parity, instantiated in some cases of value incomparability (also called 'incommensurability'). The parity view is that in at least some evaluative comparisons between two options, neither is better than the other, and they are not equally good, but the options are nevertheless comparable: they are on a par. Parity is a fourth comparative relation, besides the 'trichotomous' three of betterness, worseness, and equality.

A central argument for this view is the Chaining Argument. ${ }^{1}$ But, I argue, it has not been noticed that the Chaining Argument illicitly trades on the vagueness of its key predicate, namely, 'is comparable with'. Chang is alert to the danger of vagueness, but her defense against it applies to only one of the two ways vagueness could undermine the argument, and there is no clear way to generalize this defense.

\footnotetext{
* I am indebted to Ruth Chang, Geoffrey Sayre-McCord, Keith Simmons, and three anonymous reviewers for this journal, for extensive discussion and comments.

1. Ruth Chang, "The Possibility of Parity," Ethics 112 (2002): 659-88, esp. sec. 2. Chang has also defended parity elsewhere. For discussion, see, e.g., Joshua Gert, "Value and Parity," Ethics 114 (2004): 492-510; and Ryan Wasserman, "Indeterminacy, Ignorance and the Possibility of Parity," Philosophical Perspectives 18 (2004): 391-403.
} 
In Section I, I sketch the terrain. In Section II, I describe the Chaining Argument. Section III shows how the Chaining Argument is structurally similar to a sorites fallacy and describes Chang's defense against this. Section IV shows that this defense is ineffective against another overlooked way in which the Chaining Argument could be a sorites. In Section V, I argue that this possibility explains the intuitive plausibility of the argument's central premise. In Section VI, I argue that Chang's defense cannot be extended to cover this possibility without question begging. Section VII concludes with an argument that no chaining-type argument is likely to be effective in defense of parity.

\section{THE TERRAIN}

In this dialectic, we are concerned with cases of trichotomous incomparability:

Trichotomous Incomparability. Two objects A and B are trichotomously incomparable just in case it is not true that $\mathrm{A}$ is better than $\mathrm{B}$, not true that $\mathrm{B}$ is better than $\mathrm{A}$, and not true that $\mathrm{A}$ and $\mathrm{B}$ are equally good.

This definition of trichotomous incomparability rules out 'epistemic' views, according to which, relative to a given comparison, it is always the case that one option is better or they are equally good, although we are frequently ignorant of which. Chang argues convincingly against such views. $^{2}$

Let's follow Chang in two ways. First, 'good' and 'better' are often placeholders for some more specific evaluative term. We will be especially concerned with the evaluative predicate 'creative' and its comparatives. Second, we use the names 'Mozart' and 'Michelangelo' somewhat stipulatively: (i) they are trichotomously incomparable, with respect to creativity; and (ii) they are the most favorable case for the parity view-if parity exists, then Mozart and Michelangelo are creatively on a par.

Following John Broome, such cases of incomparability are usefully represented as a standard configuration. ${ }^{3}$ Figure 1 shows how we might represent the comparison of various sculptors to Mozart, with respect to artistic creativity. Here Mozart is the 'standard' in question, against which the sculptors will be compared. The arrow indicates sculptors of increasing creativity; the dashed lines indicate that the nature of the zonal boundaries is unspecified. For clarity, I have included a region of (perhaps

2. See the discussion at Chang, "The Possibility of Parity," 668ff., and the works cited there.

3. John Broome, "Is Incommensurability Vagueness?" in Incommensurability, Incomparability, and Practical Reason, ed. Ruth Chang (Cambridge, MA: Harvard University Press, 1997), 67-89. 


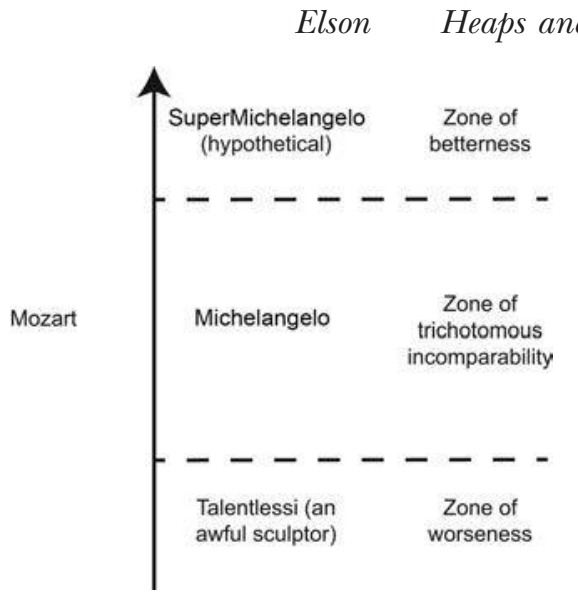

FIG. 1.-Standard configuration with trichotomous incomparability

merely possible) sculptors who are more creative than Mozart. Finally, the precise relative positions of Mozart and Michelangelo should be ignored; they are artifacts of diagramming.

The question at hand is, what's going on in the zone of trichotomous incomparability? It is natural to think that if A and B are trichotomously incomparable, then they are incomparable full stop:

Hard Incomparabilism. In cases of trichotomous incomparability, it is false that A is better than B and false that B is better than A and false that $\mathrm{A}$ and $\mathrm{B}$ are equally good. In virtue of this, $\mathrm{A}$ and $\mathrm{B}$ are not evaluatively comparable. ${ }^{4}$

To say that A and B are evaluatively comparable is to say that there is a true comparison that holds between them. ${ }^{5}$ If Hard Incomparabilism is right, then Mozart and Michelangelo are trichotomously incomparable and incomparable full stop (see fig. 2). After all, what other comparison could hold? This intuition is what grounds the Trichotomy Thesis:

Trichotomy Thesis. "[If] two items A and B are evaluatively comparable, then $\mathrm{A}$ must be better or worse than $\mathrm{B}$, or $\mathrm{A}$ and $\mathrm{B}$ must be equally good." ${ }^{\prime \prime}$

If the Trichotomy Thesis is right, then the only such relations are the classic three. Chang denies this trichotomous orthodoxy:

4. For a classic presentation, see Joseph Raz, "Value Incommensurability: Some Preliminaries," Proceedings of the Aristotelian Society, n.s., 86 (1985-86): 117-34.

5. In particular, 'comparable' does not mean more or less equal, as sometimes in everyday use.

6. Chang, "The Possibility of Parity," 660. 


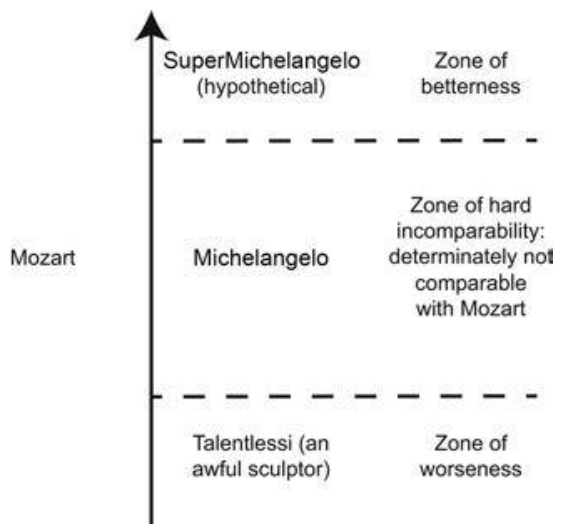

FIG. 2.-Hard Incomparabilism

Parity View. There are some cases of trichotomous incomparability which are nevertheless cases of evaluative comparability. In these cases, the trichotomous relations determinately do not hold, and the fourth relation of parity determinately holds.

On this view, parity is not derivative or otherwise second class. It is as respectable as betterness, worseness, and equality. So even though Mozart and Michelangelo are trichotomously incomparable, they are comparable. There is a fourth comparative which applies. Mozart and Michelangelo are on a par (see fig. 3). So the locus of disagreement between Hard Incomparabilism and parity amounts to this: Is there such a Mozart and Michelangelo, comparable yet not trichotomously comparable? Hard Incomparabilism stands or falls with the Trichotomy Thesis. Trichotomy is true just in case there is no such pair, and in that case Hard Incomparabilism is the right view.

Ruth Chang offers an existence proof for parity and thus an argument that Trichotomy is false; in terms of our stipulations, she gives an argument that Mozart and Michelangelo are on a par. This is the Chaining Argument, and it is the topic of this article.

\section{THE CHAINING ARGUMENT}

The Chaining Argument has two uncontroversial premises and two crucial and nonobvious premises. The first uncontroversial premise follows from our stipulations:

(CA1) Mozart and Michelangelo are trichotomously incomparable: it's not true that Mozart is more creative and not true 


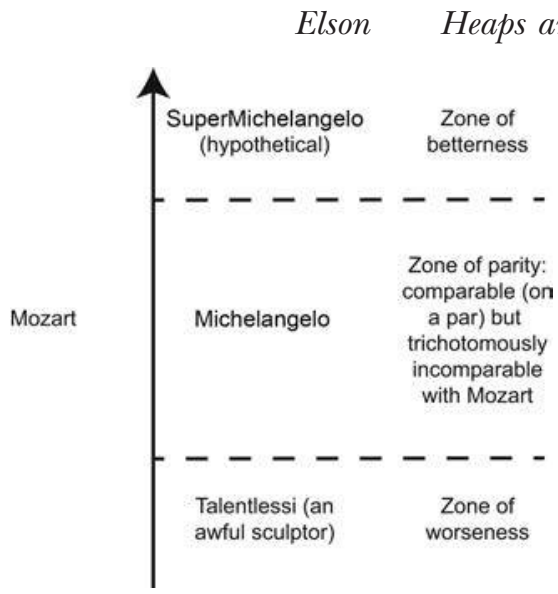

FIG. 3.-Parity

that Michelangelo is more creative and not true that they are equally creative. ${ }^{7}$

The second uncontroversial premise is that Mozart is comparable with Talentlessi, an awful sculptor-Mozart is much better. ${ }^{8}$ Let's call Talentlessi S0 (sculptor zero), and Michelangelo Sn (sculptor $n$ ) for reasons that will shortly become clear. Then:

(CA2) Mozart is comparable with S0.

The nonobvious premises are the Existence Claim and the Small Unidimensional Difference Principle.

The Existence Claim is that Talentlessi is connected to Michelangelo via a series of "small unidimensional differences." By repeatedly improving Talentlessi in single small ways, we could make him as creative as Michelangelo. Put another way, there exists a chain of (in most cases) merely possible sculptors S0 (Talentlessi), S1 (a sculptor slightly more creative in one respect than $\mathrm{S} 0$ ), S2, . . S $n$ (Michelangelo) such that between any two adjacent members of the chain there is only a small unidimensional quality difference. Let's extend our numbering in the obvious way:

(CA3) Existence Claim. The chain $\mathrm{S} 0, \mathrm{~S} 1, \ldots, \mathrm{S} n$ exists, and between any two adjacent members there is just a small unidimensional difference.

7. Remember that we are ruling out epistemicism and using these names somewhat stipulatively.

8. See Chang, "The Possibility of Parity," 672 n. 18.

9. Ibid., 673-74. 
This premise could be denied, but it is not our focus here. For our purposes, the next premise is crucial. This is the Small Unidimensional Difference Principle, which has it that these small unidimensional differences preserve comparability, at least in this most favorable case: "between two evaluatively very different items, a small unidimensional difference cannot trigger incomparability where before there was comparability. In other words, for items that bear very different respects of the covering consideration, incomparability between them cannot be a matter of some small difference in one of the respects borne such that without this small difference the items would be comparable." ${ }^{10}$ In this case, the Difference Principle claims that if Mozart is comparable with one member of the chain, then he is comparable with adjacent members of the chain:

(CA4) Small Unidimensional Difference Principle. If Mozart is comparable with some $\mathrm{S} k$ in the chain, then if there are $\mathrm{S}(k-1)$ and $\mathrm{S}(k+1)$ in the chain, then Mozart is comparable with $\mathrm{S}(k-1)$ and $\mathrm{S}(k+1)$.

We are ready to state the rest of the Chaining Argument:

(CA5) Mozart is comparable with $\mathrm{S} n$ (Michelangelo). (CA2, CA3, CA4, and $n$ applications of modus ponens.)

(CA6) Mozart is comparable but not trichotomously comparable with Michelangelo. (CA5 and CA1.)

(CA7) So the Trichotomy Thesis and Hard Incomparabilism are false, and a fourth comparison-stipulatively named 'parity'-holds between Mozart and Michelangelo. (CA6.)

The structure of this argument is clear. Since Mozart is comparable with Talentlessi, and there exists a chain of the right sort from Talentlessi to Michelangelo, we may repeatedly apply the Difference Principle and show that Mozart is comparable with Michelangelo. But none of the original three comparative relations applies, so Mozart and Michelangelo are comparable in some fourth way-parity. If this argument is sound, then Hard Incomparabilism, which denies that Mozart and Michelangelo are comparable, is false.

\section{IS THE CHAINING ARGUMENT A SORITES?}

The Chaining Argument resembles 'classic' cases of sorites reasoning:

(R1) Someone with $£ 1$ is not rich. (Premise.)

(R2) If someone with $£ k$ is not rich, then someone with $£(k+1)$ is not rich. (Premise.)

10. Ibid., 674 . 
(R3) So, someone with a million pounds is not rich. (R1, R2, 999,999 instances of modus ponens.)

Call this the 'rich man argument'. It is not a sound argument for its conclusion but is a sorites fallacy. Given this structural similarity, we should worry that the Chaining Argument is also a sorites.

Chang rightly notes that not all arguments with the form of a sorites are sorites. Mathematical induction is valid (one hopes), but it has a similar form to the sorites. So is the Chaining Argument more like the rich man or more like mathematical induction, in the relevant respects? The structural similarity between the cases introduces a burden that must be discharged: Why should we not think that the Chaining Argument is a sorites? ${ }^{11}$

Sorites fallacies turn on the vagueness of the predicates involved. In particular, it is the vagueness in 'is rich' that renders the rich man argument fallacious and a lack of corresponding vagueness that renders mathematical induction respectable. So showing that there is no vagueness present is an effective means of discharging this burden.

Chang adopts this strategy to mitigate the worry that the arguments for parity "look suspiciously like sorites arguments." In particular, she argues that "if [the arguments] did turn on the vagueness of these comparatives, the cases at issue [i.e., cases of trichotomous incomparability] would not be examples of some fourth value relation but rather borderline cases of one of the traditional trichotomy of relations." ${ }^{12}$ Chang offers several 'direct' arguments that trichotomous incomparability is not borderlineness, and so the consequent of this implication is false. She rejects the semantic indeterminist view of incomparability:

Semantic Indeterminism. It is determinate that either Mozart is better than Michelangelo or that Michelangelo is better than Mozart or that they are equally good. However, it is indeterminate which comparison applies. ${ }^{13}$

On the semantic indeterminist view, 'Michelangelo is incomparable with Mozart' is true just in case it is indeterminate which of the comparisons better/worse/equality applies between Michelangelo and Mozart. Depending on one's account of indeterminacy, it may be deter-

11. I'm grateful to an anonymous referee for pointing out several infelicities and unclarities in this part of an earlier version of the article.

12. Chang, "The Possibility of Parity," 665.

13. A neat defense of this view (but which I think ultimately fails) is Broome, "Is Incommensurability Vagueness?" I criticize Broome's argument in my "Borderline Cases and the Collapsing Principle," Utilitas (forthcoming), doi:http://dx.doi.org/10.1017/S095382081 300023X. I defend a version of the view in my "Incommensurability as Comparative Borderlineness" (unpublished manuscript, University of North Carolina, Chapel Hill, 2013). 
minate that one of them applies but indeterminate which. To say that they are incomparable is to make a metalinguistic claim: ${ }^{14}$ that Michelangelo is a borderline case of the comparative predicate 'is more creative than Mozart'. ${ }^{15}$

This indeterminacy might be grounded in indeterminacy in how the features relevant to 'is more creative' are to be weighed: on some acceptable weightings, Mozart is better; on some, Michelangelo is better; perhaps on a few, Mozart and Michelangelo are equally good. Then it will be indeterminate which of the comparisons applies (see fig. 4) ${ }^{16}$ As I mentioned, Chang offers several arguments against Semantic Indeterminism. ${ }^{17}$ The claim is that if we can show that Semantic Indeterminism is false, then the predicates in question are not vague in a way that would undermine the Chaining Argument.

So Chang offers a three-stage response to the worry that the Chaining Argument might turn on vagueness: (1) if the argument is a sorites, then the predicate involved is vague; (2) if the predicate involved is vague, then Semantic Indeterminism is true; and (3) the direct arguments refute Semantic Indeterminism. Item 1 is unproblematic, and we may accept 3 for the sake of argument. But in the next section, we'll see that 2 is false: Semantic Indeterminism is not the only way that 'is comparable with Mozart' could be vague.

\section{FUZZY HARD INCOMPARABILISM}

Let's look again at Hard Incomparabilism, which has it that Michelangelo occupies a zone of determinate incomparability with Mozart. That view does not specify the nature of the boundaries of that zone. In particular, that zone could have vague boundaries. Such a view would also posit vagueness, but it would be quite distinct from Semantic Indeterminism:

Fuzzy Hard Incomparabilism. Hard Incomparabilism is correct. There are some sculptors who are determinately incomparable with Mozart and some who are determinately comparable with Mozart.

14. Or an epistemic one, or a metaphysical one, depending on one's view of vagueness.

15. And, depending on the case, of its 'less creative' and 'equally creative' counterparts.

16. Remember that we are setting aside epistemicism as an account of trichotomous incomparability. Note that one could combine Semantic Indeterminism with an epistemicist view of vagueness; I would also count this as a form of Semantic Indeterminism, albeit a terminologically odd one. The core claim of Semantic Indeterminism is that incomparability is borderlineness; this is compatible with an ignorance analysis of borderline cases of vague predicates.

17. Chang, "The Possibility of Parity," 682ff. These arguments are incisive and are among the central challenges to my own semantic indeterminist view. I respond to them in my "Incommensurability as Comparative Borderlineness." 


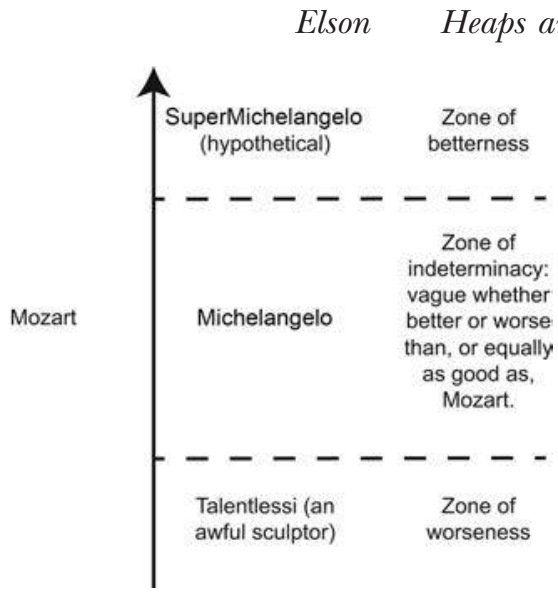

FIG. 4.-Semantic Indeterminism

But the boundary between the comparable and the incomparable is vague. Some sculptors lie on the vague border between being incomparable with Mozart and worse than Mozart. They are borderline comparable with Mozart. ${ }^{18}$

On this view, there is no determinate number of distinct small unidimensional improvements required before the improved sculptor is incomparable with Mozart (see fig. 5). The answer to that question is vague, just like the answer to the question 'how many pounds does a pauper need to become rich?' is (see fig. 6). But just as this is compatible with there being many amounts of money $n$ such that having $£ n$ does make one determinately rich, so there are many sculptors at the 'upper end of the chain' such that having been improved $n$ times does make $\mathrm{S} n$ determinately incomparable with Mozart. ${ }^{19}$ This shows that 2 is false: even though this view is not Semantic Indeterminism, the crucial predicate 'is comparable with Mozart' is vague.

Even if Chang's direct arguments are effective against Semantic Indeterminism, they do nothing to rule out this view. The target of those arguments is the possibility in which cases of trichotomous incompara-

18. As two anonymous referees have rightly pointed out to me, it is slightly tendentious to claim that if the boundary is vague, then there are borderline cases. Such talk adds clarity but should officially be interpreted in terms of a vague boundary. My argument does not rely on borderline cases: it is not borderline cases but tolerance (discussed below) that brings the possibility of sorites fallacy. As an autobiographical note, I suspect that borderlineness is central to vagueness, but that is admittedly controversial.

19. It might be worried that the two cases are somewhat disanalogous because 'is rich' is monadic, whereas 'is comparable with' is relational. But this is not a problem: the predicate in the Chaining Argument is 'is comparable with Mozart', which is monadic. 


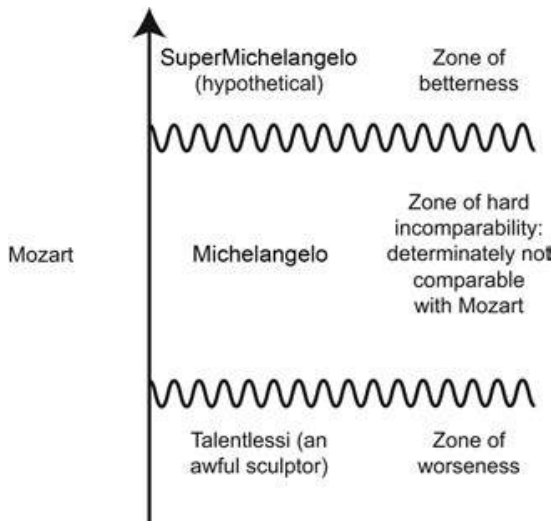

Fig. 5.-Fuzzy Hard Incomparabilism (wavy lines indicate vagueness)

bility "would not be examples of some fourth value relation but rather borderline cases of one of the traditional trichotomy of relations." ${ }^{20} \mathrm{In}$ other words, the arguments are against the claim that cases of trichotomous incomparability are borderline applications of a vague predicate (or several). They aim to show that there are significant differences between cases of incomparability like that of Mozart and Michelangelo with respect to creativity and vague-borderline cases like those of "is balder than'. But Fuzzy Hard Incomparabilism does not claim that cases of incomparability are borderline cases; its central claims are that cases of incomparability are those in which the three trichotomous comparisons betterness/worseness/equality determinately fail to apply and that 'is incomparable with Mozart' has vague boundaries.

In slogan form: Semantic Indeterminism is the claim that incomparability is a phenomenon of vagueness - to be incomparable with Mozart is to be a borderline case of the three trichotomous comparisons. Fuzzy Hard Incomparabilism denies this. On this view, to be incomparable with Mozart is for each of these comparisons to be false. But the predicate 'is incomparable with Mozart' has vague boundaries, just as 'is a heap' and 'is tall' do. Since this view is inconsistent with Semantic Indeterminism, arguments against the semantic account — such as Chang's direct arguments - do not undermine Fuzzy Hard Incomparabilism.

Now, we can see how Fuzzy Hard Incomparabilism would render the Chaining Argument a sorites. The point here is twofold. First, it is admitted by all that the Chaining Argument is structurally similar to a sorites and that this introduces a burden of showing that it is not a fallacy. This was to be met by showing that the predicate in question is

20. Chang, "The Possibility of Parity," 665. 


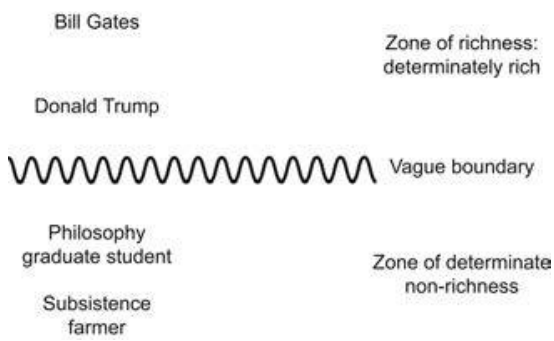

FIG. 6.-Vague boundary of 'is rich' (wavy line indicates vagueness)

not vague, but that standard has not been met: vagueness has not been ruled out.

But I also wish to make a stronger claim. The vagueness in Fuzzy Hard Incomparabilism means that the Chaining Argument is relevantly similar to that which grounds other sorites paradoxes. Sorites fallacies typically trade on the sort of vague boundaries that Fuzzy Hard Incomparabilism posits. As we have seen, the rich man argument illicitly trades on the vagueness of the predicate involved ('is rich'). Consider other classic sorites arguments, such as those involving 'is tall', 'is a heap', or 'is bald', for example. Each of these is grounded in the fact that the predicate involved has vague boundaries, which is what Fuzzy Hard Incomparabilism claims for 'is comparable with Mozart'.

Let's focus on the rich man. In response to worries that this argument is a sorites fallacy, we might (correctly) claim that these worries could be dispelled by showing that 'is rich' is not vague. But to carry this plan out, it would not do merely to show that a semantic indeterminist account of richness (according to which to be rich is to be a borderline case of some other predicate) is false. We would need to show is that 'is rich' does not have vague boundaries. It is the analogue of this latter claim which would undermine Fuzzy Hard Incomparabilism and which Chang's direct arguments do not establish.

The point is not just that there are two ways that is comparable with' could be vague-Semantic Indeterminism and Fuzzy Hard Incomparabilism - and that Chang's direct arguments rule out only one of them. The kind of vagueness which is not ruled out (that which is characteristic of Fuzzy Hard Incomparabilism) grounds the most common instances of the sorites paradox, including the original: 'is a heap' has vague boundaries, but to be a heap is not to be a borderline case of some other predicate. The direct arguments not only fail to rule out half of the sources of vagueness; they fail to rule out the more threatening half. In the next section, I present another reason to think the Chaining Argument is a sorites - seeing it as a sorites explains the undeniable plausibility of its key premise. 


\section{AN ERROR THEORY FOR THE DIFFERENCE PRINCIPLE}

Fuzzy Hard Incomparabilism can also explain the plausibility of the Difference Principle. Here are some quotes from Chang's defense of that principle:

Now we bring to bear a key intuition ... between two evaluatively very different items, a small unidimensional difference cannot trigger incomparability where before there was comparability ... a small unidimensional difference just does not seem powerful enough to effect a switch from two such items being comparable to their being incomparable. ${ }^{21}$

The Small Unidimensional Difference Principle has deep intuitive appeal. It is just plain hard to believe of two evaluatively very different but by hypothesis comparable items that making a small unidimensional improvement or detraction in one of them can thereby effect a switch from the items' being comparable to their being incomparable. ${ }^{22}$

How can changing one of the careers ever so slightly in a single respect change the relation between the careers such that before there was an evaluative difference and now there is none-not even a zero difference? ${ }^{23}$

This consideration is by no means decisive, but this is noticeably similar to how one might motivate tolerance principles in the case of vague predicates: How could adding just $£ 1$ make a nonrich man rich? It's just hard to believe; the addition of just $£ 1$ doesn't seem powerful enough to effect such a change. Chang is correct that the Difference Principle has 'deep intuitive appeal', but if there is a boundary between the comparable and the incomparable zones (i.e., if some version of Hard Incomparabilism is right), then the Difference Principle can be read as a statement that this boundary has vagueness.

I think that the best diagnosis of the principle's intuitive appeal is that it is a tolerance principle for a vague predicate. As Crispin Wright has argued, tolerance ("a notion of a degree of change too small to make any difference, as it were") is a key characteristic of vague predicates that are vulnerable to the sorites paradox. ${ }^{24}$ One millimeter couldn't make a short man not short, one hair couldn't make a bald child not

21. Ibid., 674 .

22. Ibid., 675 .

23. Ibid., 676 .

24. Crispin Wright, "On the Coherence of Vague Predicates," Synthese 30 (1975): 32565 , quote on 333 
bald, and so on. In the same way as the vagueness of 'rich' can make 'if a man with $£ n$ is rich, then a man with $£(n+1)$ is rich' intuitively compelling, so the vagueness of 'comparable' on Fuzzy Hard Incomparabilism would be expected to make 'if $\mathrm{S} n$ is comparable with Mozart, then $\mathrm{S}(n+1)$ is comparable with Mozart' compelling.

The key point is that the principle would appear to be correct, even if it were just an artifact of the vague boundary of 'is comparable with Mozart'. If Fuzzy Hard Incomparabilism were correct, we would expect a tolerance principle identical to the Difference Principle to be intuitively overwhelming. The Difference Principle is intuitively plausible. But this intuitive plausibility adds to the suspicion that is comparable with Mozart' might have vague boundaries and that Fuzzy Hard Incomparabilism might be correct.

\section{CAN CHANG'S STRATEGY BE GENERALIZED?}

We've seen that the possibility of Fuzzy Hard Incomparabilism is one way that the Chaining Argument could trade on the vagueness of 'is comparable with Mozart', that this could explain the plausibility of the Difference Principle, and that arguments against Semantic Indeterminism are ineffective against this. But what about an adaptation of Chang's strategy — showing that 'is comparable with Mozart' also lacks this sort of vagueness? This would be to acknowledge that there is a burden of showing that 'is comparable with Mozart' does not have vague boundaries and to try to meet it. This is not promising. It would be to show that the Hard Incomparabilism in question is not fuzzy, but sharp:

Sharp Hard Incomparabilism. Hard Incomparabilism is correct. Michelangelo is incomparable with Mozart. Every object is either determinately comparable with Mozart or determinately incomparable with Mozart. There are no 'borderline-comparable' objects. The predicate 'is comparable with Mozart' does not have vague boundaries.

On this view, there would be some number $k$ of small unidimensional improvements to Talentlessi, such that a sculptor with $k$ improvements is worse than-and therefore comparable with-Mozart, but a sculptor with $(k+1)$ is not better than, worse than, or as good as Mozart and is therefore incomparable with him (see fig. 7). Such a strategy could show that the Chaining Argument is valid: the Chaining Argument is certainly valid against Sharp Hard Incomparabilism. But this would be at the cost of completely defanging the argument. The crucial premise of the Chaining Argument is the Small Unidimensional Difference Principle, which says that for every $i$, if $\mathrm{S} i$ is comparable with Mozart, then $\mathrm{S}(i+1)$ and 


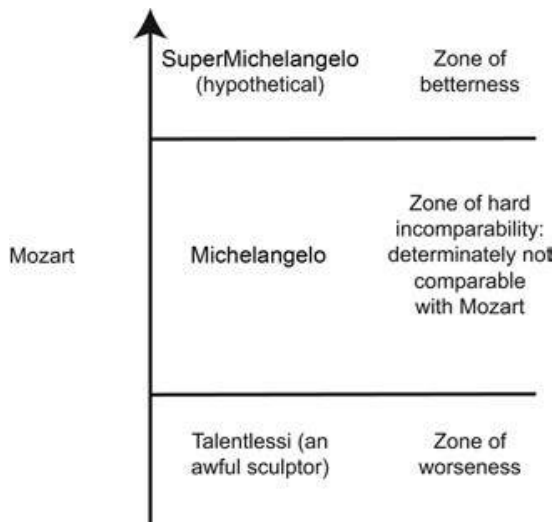

FIG. 7.-Sharp Hard Incomparabilism (horizontal lines indicate lack of vagueness)

$\mathrm{S}(i-1)$ are comparable with Mozart. ${ }^{25}$ The Difference Principle denies that 'is comparable with Mozart' could be sharply bounded. But the claim that there is such a sharp boundary is what is absolutely distinctive of Sharp Hard Incomparabilism.

If the Difference Principle is true, then Sharp Hard Incomparabilism is false. The real work of refuting Sharp Hard Incomparabilism would be in establishing the Difference Principle. But once that had been done, the Chaining Argument would be otiose since it would add no additional weight to a rejection of Sharp Hard Incomparabilism. The Chaining Argument cannot be dialectically effective against Sharp Hard Incomparabilism since a premise of the argument - the Difference Principle - is incompatible with that view.

\section{CONCLUSION}

Hard Incomparabilism, which is the target of the Chaining Argument, must posit either vague or sharp boundaries to the zone of incomparability. If the boundaries are vague (Fuzzy Hard Incomparabilism), then the Chaining Argument has sorites structure and uses the vague predicate 'is comparable with Mozart'. If they are not vague (Sharp Hard Incomparabilism), then the Chaining Argument is not a sorites, but its key premise - the Difference Principle-immediately falsifies the target view, so the real work is done in defending the Difference Principle.

The apparent soundness of the Chaining Argument relies on eliding the distinction between the two versions of Hard Incomparabilism.

25. Officially, we should add an 'if they exist on the chain' qualifier here, to deal with the endpoints of the chain, if there are any. 
But once this distinction is seen, it provides a dilemma for that argument. Either it is a sorites fallacy (or should be treated as one because the burden of showing that it is not has not been met) or it contributes nothing, dialectically, beyond the defense of the Difference Principle.

The Chaining Argument cannot fulfill its dialectical role of showing that on premises that everyone should accept-Mozart-Talentlessi comparability, the Existence Claim, the Difference Principle, and the trichotomous incomparability of Mozart and Michelangelo-there is a good argument for parity. The class of opponents against whom the Chaining Argument was directed-hard incomparabilists-can neatly explain away the apparent plausibility of its most crucial premise, the Difference Principle.

We would accept the argument's key premise - the Difference Principle - only if we were already convinced of the falsity of both forms of Hard Incomparabilism. If we were not convinced of the falsity of Fuzzy Hard Incomparabilism, we would worry that the Difference Principle might be an artifact of the vagueness of 'is incomparable with Mozart'. If we were not convinced of the falsity of Sharp Hard Incomparabilism, we would worry that the Difference Principle is question begging. So the Chaining Argument cannot offer any additional reasons to deny Hard Incomparabilism.

For all that, I have not shown that parity is false or even that the Chaining Argument is definitely an instance of the paradox of the sorites. To show the latter, one needs the equivalent of the claim that "of course someone with $£ 1$ million is rich, so the argument must have gone wrong somewhere'. Here that would be a claim that 'of course Mozart and Michelangelo are not comparable...', which is obviously not available in the current dialectic.

But I do not think the Chaining Argument can be rescued. Chang's paper is admirably 'modular': she carves up the space of views, and rejects each alternative, so that only parity is left standing. The Chaining Argument's job is to refute the Trichotomy Thesis and thereby Hard Incomparabilism. But the current discussion shows that the Chaining Argument cannot do this job. This job could be done by another argument, which would, together with the arguments against other views, also provide an existence proof for parity. But it would not be the Chaining Argument. 